.

\title{
Oclusão Percutânea de Forame Oval Patente em Paciente Portadora de Síndrome de Platipneia- Ortodeóxia com Aorta Ectásica: Relato de Caso
}

\author{
Patent Foramen Ovale in a Patient with Platypnea-Orthodeoxia \\ Syndrome with Ectasic Aorta: \\ A Case Report
}

Joberto Pinheiro Sena ${ }^{1}$, Adriano Dias Dourado Oliveira ${ }^{1}$, Hilana Renata Moreira Araújo ${ }^{1}$

${ }^{1}$ Serviço de Cardiologia do Hospital Santa Izabel; Salvador, Bahia, Brasil

Correspondence addresses:

Dr. Joberto Pinheiro Sena

jobertosena@terra.com.br

Received: April 19, 2019

Revised: May 31, 2019

Accepted: June 9, 2019

Published: June 28, 2019

Data Availability Statement: All relevant data are within the paper and its Supporting Information files.

Funding: This work was the result of authors' initiative. There was no support of research or publication funds.

Competing interests: The authors have declared that no competing interests exist.

Copyright

(C) 2019 by Santa Casa de Misericórdia da Bahia. All rights reserved.

ISSN: 2526-5563

\begin{abstract}
Descrevemos o caso de uma paciente que, ao assumir posições de ortostatismo, apresentava hipoxemia e dispneia. Os exames iniciais identificaram apenas a presença de ectasia da aorta, sem, no entanto, justificar o quadro. Realizado ecocardiograma transesofágico, confirmou-se o diagnóstico de forame oval patente com intensificação do fluxo direita para esquerda com a mudança do décubito. A associação dessas manifestações a uma etiologia incomum, o shunt intracardíaco, caracterizou o diagnóstico da síndrome de platipneia-ortodeóxia. Realizada oclusão percutânea do forame oval patente com melhora completa dos sintomas, evoluindo estável 7 meses após o procedimento e sem queixas. Palavras-chave: Síndrome de Platipneia-Ortodeóxia; Aneurisma Aórtico; Ocluão Percutânea de Forame Oval Patente.
\end{abstract}

We describe a case of a patient who presented hypoxemia and dyspnea when was in orthostatic position. Initial examinations identified the presence of aortic ectasia, which did not justify the patient's condition. A transesophageal echocardiogram was performed, confirming the diagnosis of patent foramen ovale with the intensification of the right to left flow with the change of the decubitus. The association of these manifestations with an unusual etiology, the intracardiac shunt, characterized the diagnosis of the platypnea-orthodeoxia syndrome. Percutaneous occlusion of the patent foramen ovale was performed with a complete improvement of the symptoms, and stable 7 months after the procedure and no complaints.

Keywords: Platypnea-Orthodeoxia Syndrome; Aortic Aneurysm; Percutaneous Occlusion of the Patent Foramen Oval.

\section{Introdução}

A síndrome de platpneia-ortodeóxia (SPO) é uma condição clínica rara caracterizada pela ocorrência de dispneia e hipoxemia significativas em ortostase, com melhora ao decúbito. A sua principal causa é a presença de shunt intracardíaco através do forame oval patente (FOP). O fechamento percutâneo do forame oval patente (FOP), procedimento já estabelecido para a prevenção da recorrência de acidente vascular encefálico (AVC), é 
o tratamento indicado nesses casos. Este relato descreve a investigação, diagnóstico e tratamento de paciente com SPO.

\section{Relato do Caso}

IVA, 67 anos, sexo feminino, circense, viúva, natural e procedente de Aracaju. Paciente hipertensa, diabética e com história de AVC isquêmico há 6 anos. Atendida com queixa de dispneia ao ficar sentada ou em pé, com melhora ao decúbito dorsal há um ano, comprometendo progressivamente as suas atividades, estando nas últimas semanas restrita ao leito e em uso de oxigênio. Apresentou alguns episódios documentados de dessaturação de oxigênio em ortostase. Estava em uso de metformina $850 \mathrm{mg}$, 2x/dia; olmesartana + hidroclorotiazida $(20+$ $12,5 \mathrm{mg}) 1 \mathrm{x} / \mathrm{dia}$. Ao exame físico estava lúcida e orientada, eupneica em decúbito e dispneica ao sentar-se, em uso de oxigênio nasal a $3 \mathrm{~L} /$ min. Oximetria de pulso de $98 \%$ em decúbito, $88 \%$ sentada e $81 \%$ em ortostase. $\mathrm{PA}=100 \mathrm{X} 60$ $\mathrm{mmHg}$ e FC $72 \mathrm{bpm}$. Peso $60 \mathrm{~kg}$, altura $1,60 \mathrm{~m}$ e IMC $24,61 \mathrm{~m}^{2}$. Murmúrio vesicular diminuído difusamente e ausência de ruídos adventícios. Precórdio calmo, ictus palpável no $5^{\circ}$ EICE, $2 \mathrm{~cm}$ a esquerda da LMC, abrangendo 2 polpas digitais. Rítmo cardíaco regular em 2 tempos, sem sopros. Ausência de turgência jugular patológica.

Radiografia de tórax evidenciou aumento do índice cardiotorácico e alongamento importante da aorta (Figura 1). Ecocardiograma transtorácico e transesofágico confirmaram a presença de forame oval patente, com shunt direito/esquerdo significante, que se acentuou à elevação do decúbito. Ventrículo esquerdo hipertrofiado, com função sistólica preservada, esquerda preservada e aorta ectasiada $(4,8 \mathrm{~cm})$.

Confirmado o diagnóstico de SPO por shunt intracardíaco, a paciente foi encaminhada para a oclusão percutânea.

Cateterismo cardíaco: pressões normais em ventrículo direito e artéria pulmonar; fluxo pulmonar preservado, ausência de malformações artério-venosas pulmonares; aorta ascendente dilatada e alongada, comprimindo ventrículo direito. Posicionado e insuflado balão de medição $24 \mathrm{~mm}$, até desaparecimento de shunt, sendo feita medida pelo ecocardiograma transesofágico. Implantada prótese Lepu Memopart PFO 30 $\mathrm{mm}$. Após manobras de segurança e avaliação do ecoardiograma dispositivo foi liberado (Figura 2). Teste de microbolhas demonstrou ausência de shunt.

Paciente manteve bom padrão respiratório, em ar ambiente, sem novos episódios de dessaturação e sem necessidade de oxigênio suplementar. Saturação de oxigênio de $98 \%$, em decúbito e ortostase, sem queixas de dispneia, evoluindo assintomática no seguimento de 7 meses após o procedimento.

\section{Discussão}

A síndrome de platpneia-ortodeóxia (SPO) é uma condição clínica rara em que ocorre hipoxemia ao se colocar em posição de ortostase e essa dessaturação promove dispneia. $\mathrm{O}$ termo platipneia-ortodeóxia foi cunhado pela primeira vez em 1969 em paciente com síndrome hepatopulmonar e apenas em $1984 \mathrm{em}$ paciente com shunt intracardíaco. A SOP tem como fisiopatologia o aumento rápido do volume de sangue insaturado na circulação arterial causado por aumento de shunt direito-esquerdo ou de defeito da ventilação-perfusão pulmonar. A sua principal etiologia é a presença de defeito anatômico cardíaco, sendo o forame oval patente (FOP) o mais frequente.

O FOP geralmente é assintomático e encontrado em até $25 \%$ da população adulta. Em situações de aumento das pressões nas câmaras direitas ou de uma reorientação do fluxo venoso em direção à face do FOP, voltada para o átrio direito, pode ocorrer shunt direito-esquerdo. Há casos em que o shunt se deve a alterações do gradiente pressórico (pressões no átrio direito maiores que no esquerdo), sendo tanto a dispneia 
Figura 1, Radiografia de tórax com aumento do índice cardiotorácico e alongamento da aorta.

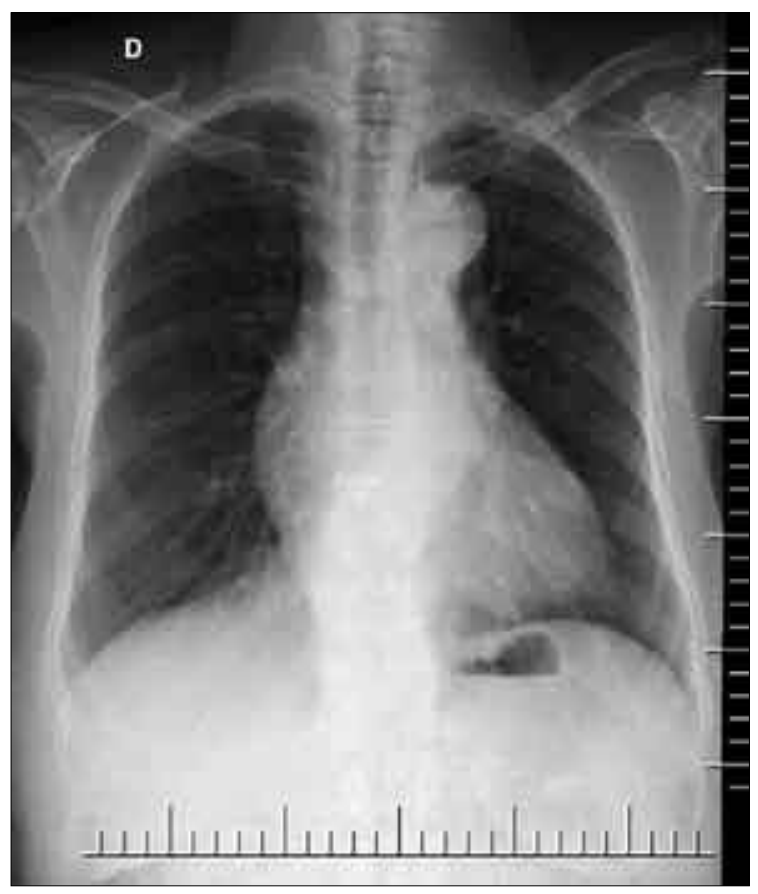

Figura 2. Implantação da prótese Lepu Memopart PFO $30 \mathrm{~mm}$.

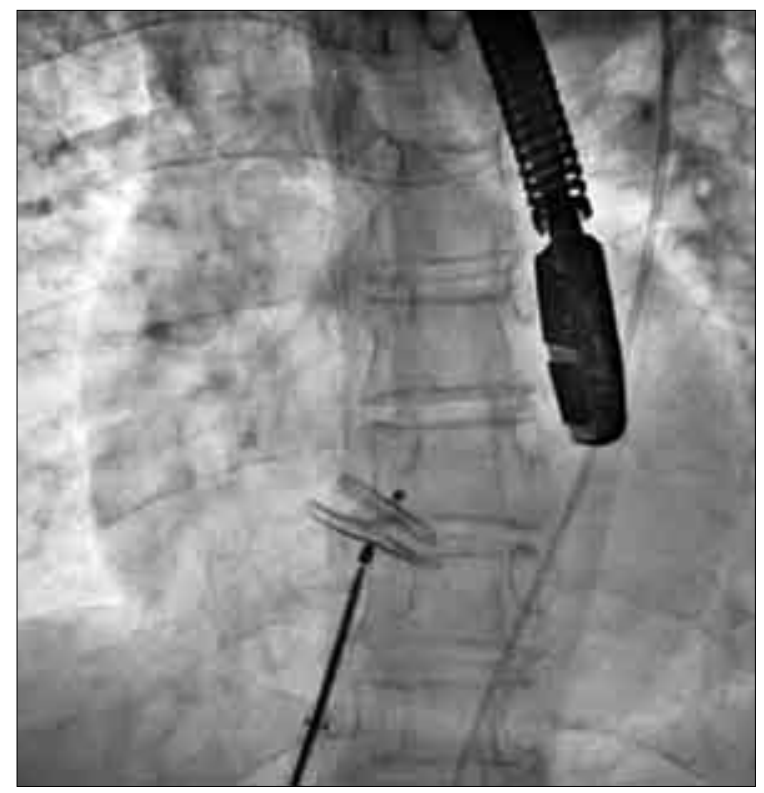

quanto a hipoxemia não influenciadas pela posição do paciente.

$\mathrm{Na}$ SPO, o shunt direito-esquerdo não está relacionado a elevações das pressões nas câmaras direitas. É necessária a existência de fatores anatômicos e/ou funcionais capazes de alterar as relações das estruturas intracardíacas, orientando o fluxo venoso preferencialmente em direção ao septo interatrial e determinando aumento do shunt pelo FOP ao se levantar. Eles podem ser congênitos (mecanismos persistentes da circulação fetal), como por exemplo a rede de Chiari e a válvula de Eustáquio proeminente, e adquiridos. Entre os fatores adquiridos, aparecem a pneumonectomia, a atelectasia, paralisia do diafragma, cifoescoliose e ectasia/aneurisma da raiz da aorta. A dilatação da aorta ascendente, com compressão do ventrículo direito, como no caso relatado, é a causa mais frequente. A associação entre a ectasia da raiz da aorta e o desenvolvimento do shunt direto-esquerdo foi descrita em diversas populações. Eicher e colaboradores ${ }^{5}$ avaliaram, retrospectivamente, uma série de 19 pacientes com a SPO por shunt intracardíaco e encontraram a presença de dilatação da raiz da aorta em $63 \%$ deles. Em $42 \%$ dos casos, não havia qualquer outra alteração toracopulmonar que pudesse justificar a síndrome. Bertaux e colaboradores ${ }^{6}$ descreveram alterações no tamanho e na mobilidade do septo interatrial determinadas pela presença de aneurisma da raiz da aorta sendo verificado que, quanto maior a dilatação da raiz da aorta, menor e mais móvel se torna o septo interatrial e que isso, na presença de FOP, permitiria e potencializaria o shunt direito-esquerdo.

As etiologias pulmonares importantes são as malformações arterio-venosas, a síndrome hepatopulmonar e a síndrome de angústia respiratória aguda (SARA), promovendo aumento do shunt intrapulmonar, ou aquelas que promovem aumento da diferença entre ventilação e perfusão, como na doença pulmonar obstrutiva crônica (DPOC) ou doença intersticial. A intoxicação pulmonar por amiodarona tem sido descrita como etiologia da SOP. Trata-se de uma síndrome muitas vezes subestimada, pois sua identificação exige suspeição clínica apurada. No caso apresentado, a paciente vinha com sintomas progressivos há 1 ano, sem diagnóstico, chegando a ficar praticamente restrita ao leito e com necessidade de oxigênio domiciliar. Por outro 
lado, o diagnóstico inicial é relativamente fácil, através da simples aferição de oximetria periférica em posições diversas. A coleta de gasometrias confirma o diagnóstico quando, por definição, existir variação da $\mathrm{PaO} 2$ e Sat.O2 superiores a $4 \mathrm{mmHg}$ e $5 \%$, respectivamente. Além disso, a presença de defeito atrial e de shunt intracardíaco direito-esquerdo deve ser pesquisado e avaliado por ecocardiograma transesofágico, com teste de microbolhas na posição supina e ortostática. A tomografia e a angiotomografia são exames fundamentais para avaliação de casos de etilogia não cardíaca, como também na pesquisa de fatores anatômicos e funcionais secundários. A avaliação da Pneumologia deve ser contemplada nos casos duvidosos ou não esclarecidos (Figura 3).

O tratamento da SPO irá depender da sua etiologia principal. Nos casos de shunt intracardíacao, há evidências mostrando segurança e eficácia tanto para o fechamento percutâneo quanto cirúrgico. A oclusão transcateter do FOP, com implante de prótese, está associada a altas taxas de sucesso e poucas complicações hospitalares e na evolução a longo prazo, como na experiência do grupo de Toronto com seguimento de 2 anos.

\section{Conclusão}

A SPO é rara, mas pode comprometer muito a qualidade de vida dos pacientes quando não diagnosticada. A oclusão percutânea transcateter do defeito do septo interatrial, com implante de prótese, por ser a causa mais frequente, é terapêutica eficaz e segura para o alívio dos sintomas nesse cenário, como no caso relatado.

\section{Referências}

1. Agrawal et al. Respiratory Medicine 2017;129:31-8.

2. Tobis JM, Abudayyeh I. J Am Coll Cardiol Intv. 2016;9(18):1939-40.

3. Vecchis R, Ariano CBC J. Clin. Med. 2016;5(85). doi: $10.3390 / \mathrm{jcm} 5100085$.

4. Shah et al. J Am Coll Cardiol Intv 2016;9:1928-38.

5. Eicher JC, Bonniaud P, Baudouin N, Petit A, Bertaux G, et al. Hypoxaemia associated with an enlarged aortic root: a new syndrome? Heart 2005;91(8):1030-5.

6. Bertaux G, Eicher JC, Petit A, Dobsák P, Wolf JE. Anotomic interaction between the aortic root and the atrial septum: a prospective echocardiographic study. J Am Soc Echocardiogr. 2007;20(4):409-14.

7. Shah AH, Osten RM, Leventhal A, Bach Y, Yoo D, et al. J Am Coll Cardiol Intv. 2016;9(18).

Figura 3. Avaliação esquemática da Pneumologia para a SOP.

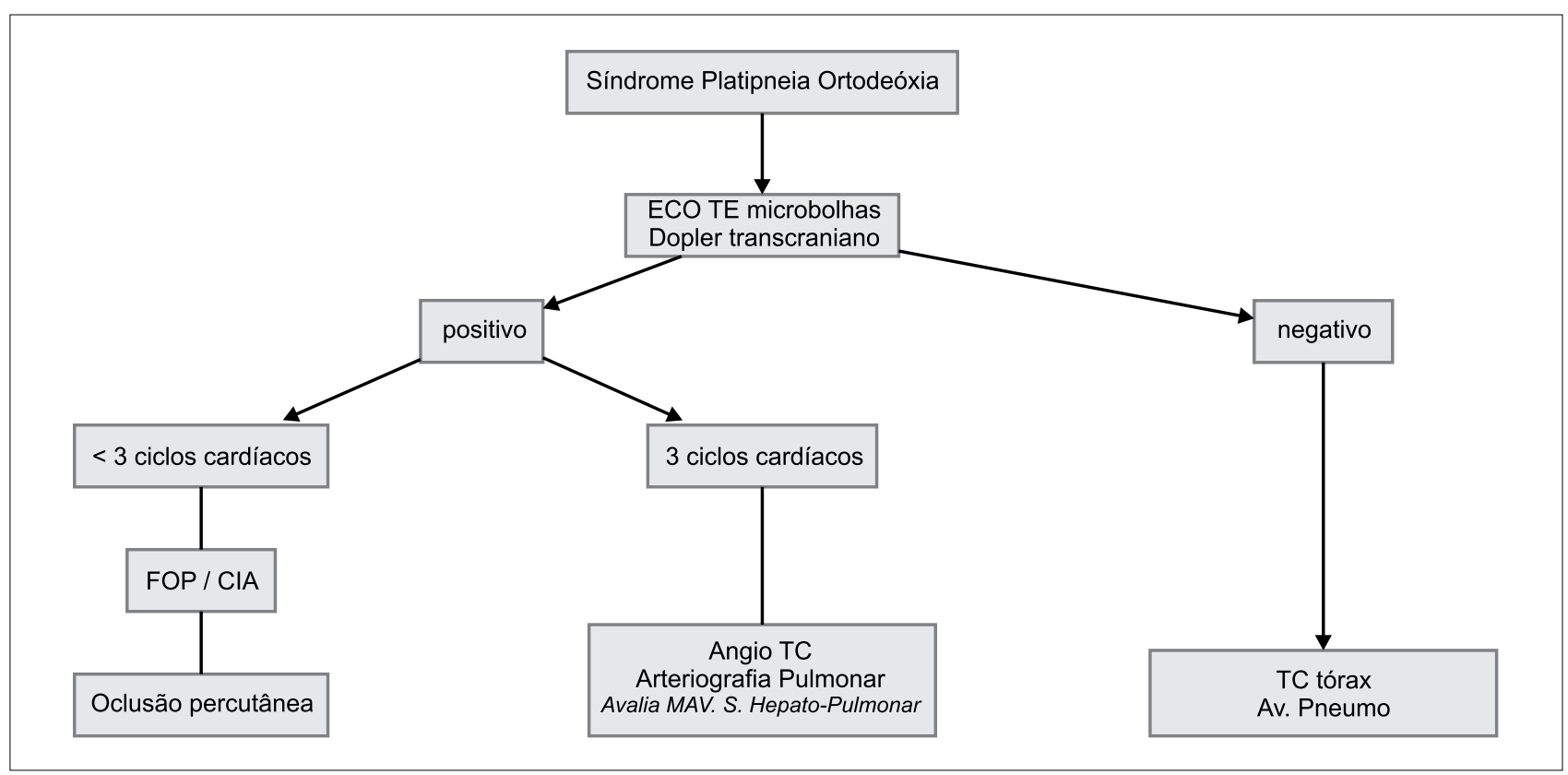

